\title{
Propuesta financiera para la creación de la ruta agro turística cafetera del municipio de Gramalote, Norte de Santander
}

\author{
Financial proposal for the creation of the \\ agro tourism coffee route of the municipality \\ of Gramalte, North of Santander
}

DOI: https://doi.org/10.17981/econcuc.41.1.2020.Econ.4

Artículo de investigación.

Fecha de recepción: 30/08/2019

Fecha de devolución: 15/11/2019

Fecha de aceptación: 25/11/2019

Fecha de publicación: 30/11/2019

\section{Johanna Milena Mogrovejo Andrade \\ Universidad Libre. \\ Cúcuta (Colombia) \\ johannam.mogrovejoa@unilibre.edu.co}

\author{
Cesar Augusto Panizo Cardona \\ Universidad Francisco de Paula Santander. \\ Cúcuta (Colombia) \\ cesaraugustopc@ufps.edu.co
}

\section{Marilyn López Echeverry \\ Universidad Libre. \\ Cúcuta (Colombia) \\ marilyn-lopeze@unilibre.edu.co}

Para citar este artículo:

Mogrovejo, J., Panizo, C. \& López, M. (2020). Propuesta financiera para la creación de la ruta agro turística cafetera del municipio de gramalote, Norte de Santander. Económicas CUC, 41(1). 81-98.

DOI: https://doi.org/10.17981/econcuc.41.1.2020.Econ.4

\section{Resumen}

En el presente artículo se analizó la viabilidad de la creación de una ruta agro turística del café para el Municipio de Gramalote (Norte de Santander, Colombia). La propuesta se realiza debido a las dificultades por ingresos que presentan las personas del campo, situación que ha creado una enorme brecha económica entre lo rural y lo urbano. En este mismo sentido, el municipio de Gramalote y sus veredas aledañas han sido tradicionalmente productoras del grano y cuenta con un rico acervo cultural y gastronómico, así como con una geografía privilegiada que la convierte en un atractivo potencial para las personas que buscan una forma de turismo diferente, basado en la tranquilidad y el aire limpio, así como la búsqueda de experiencias nuevas. Igualmente, la declaración del paisaje cultural cafetero, por la Unesco, como patrimonio de la humanidad, ha contribuido a que muchos turistas, especialmente los extranjeros, se interesen por este tipo de actividad. La investigación tuvo como objetivo general identificar las apuestas competitivas para la ruta agro turística del sector cafetero del municipio de Gramalote Norte de Santander, concluyendo que es viable desde el punto de vista económico, y los residentes de las zonas están interesados en la posibilidad de su creación debido a la posibilidad de convertirse en una excelente fuente de ingresos, permitiéndoles mejorar su calidad de vida.

Palabras clave: Agroturismo; café; cultura; paisaje; ruta; turismo

\section{Abstract}

This article analyzed the feasibility of creating an agro-tourist coffee route for the municipality of Gramalote (Norte de Santander, Colombia). The proposal is made due to the income difficulties of the rural people, a situation that has created an enormous economic gap between the rural and urban areas. In this same sense, the municipality of Gramalote and its surrounding villages have traditionally been producers of the grain and have a rich cultural and gastronomic heritage, as well as a privileged geography that makes it a potential attraction for people seeking a different form of tourism, based on tranquility and clean air, as well as the search for new experiences. Likewise, the declaration of the coffee-growing cultural landscape by UNESCO as a world heritage site has contributed to the interest of many tourists, especially foreigners, in this type of activity. The general objective of the research was to identify the competitive bets for the agrotourist route of the coffee sector in the municipality of Gramalote Norte de Santander, concluding that it is economically viable, and the residents of the areas are interested in the possibility of its creation due to the possibility of becoming an excellent source of income, allowing them to improve their quality of life.

Keywords: Agrotourism; coffee; culture; landscape; route; tourism 


\section{INTRODUCCIÓN}

Son muchas las necesidades que presentan las zonas rurales en América Latina, entre los que se pueden mencionar, fundamentalmente los que tienen que ver con la pobreza. Esta es una problemática histórica que proviene de la colonia, debido a la distribución no equitativa de las tierras, al concentrarse la gran mayoría de estos territorios en pocas manos, fenómeno comúnmente conocido como latifundismo. Estas concentraciones de tierras han obligado a muchos habitantes del sector rural a trabajar la tierra con el único incentivo de alcanzar los ingresos necesarios para la supervivencia. En este sentido Kay (2007) considera:

Las causas principales de la pobreza rural son estructurales y se relacionan con la distribución dispareja de tierras y la desigualdad del sistema de poder. El acceso al capital, a la tecnología y a los mercados, así como a los sistemas de conocimiento e información, se ha vuelto cada vez más importante para determinar el éxito en una empresa agrícola (p. 69).

De acuerdo con lo apuntado por el autor, son diversas las causas que contribuyen a los altos niveles de concentración de pobreza en el campo, especialmente la dependencia de los ingresos del sector sólo a la industria agrícola. En este orden de ideas, es relevante hacer referencia a la importancia que tiene la diversificación de las actividades rurales. "Las políticas que promuevan actividades rurales no agrícolas también pueden ayudar a reducir la pobreza rural, pero esto no debe llevarse a cabo a costa de políticas que promuevan el desarrollo agrícola” (Kay, 2007, p. 69; Pérez, Chumaceiro y Acosta, 2019). Esto implica que se debe propender por la creación de otros tipos de actividades que contribuyan a diversificar el ingreso de las zonas rurales, sin descuidar la actividad principal del agro, es decir, la producción agropecuaria.

El campo colombiano no es ajeno a este fenómeno, que pudo ser caracterizado de forma más precisa durante el Censo que se desarrolló en el país durante el año 2018, donde los censistas recorrieron las "113 millones de hectáreas de lo que se conoce como el área rural dispersa, es decir, lo que va más allá de las cabeceras y los centros poblados" (Semana, 2015). Los resultados encontrados no son nada halagadores, y se pudo verificar que la situación es mucho más compleja de lo que se creía, puesto que las brechas de desigualdad son profundas. De acuerdo con la revista Semana (2015) se encontró que para el año 2014, el $20 \%$ de la población entre los 5 y 16 años no asistía a ninguna institución educativa. También se observó como el 72,6 \% de los jóvenes entre 17 y 24 años no tiene acceso a la educación y el 11,5 \% de la población campesina mayor de 15 años son analfabetas.

Pero la dificultad no solo es en el aspecto educativo. De acuerdo el citado artículo, el censo reveló que "el índice de pobreza multidimensional en el campo es del 44,7 por ciento, el doble del registro total nacional, que para 2014 estaba en 21,9 por ciento y casi tres veces el urbano que se ubicaba en 15,4 por ciento" (Semana, 2015). Esto hace pensar en la necesidad urgente de movilizar recursos y esfuerzos 
para la diversificación de las actividades rurales, siendo el turismo agrícola uno de los sectores que más fuerza ha ido tomando en los últimos tiempos. Al respecto Pérez (2010) afirma:

Uno de los principales sistemas productivos alternativos rurales es el Turismo Rural (TR), que despliega una serie de servicios y productos generadores de ingresos adicionales o ingresos rurales no agrícolas (IRNA), por lo que se presenta como opción complementaria de la actividad agrícola, que gana competitividad cuando es la familia rural la emprendedora, trayendo de la mano valores territoriales materiales e inmateriales ( $p$. 508).

La fortaleza de esta alternativa está en el auge que ha ido tomando el agroturismo, debido a la búsqueda de alternativas por parte de las personas de las ciudades para descansar, pero sobre todo para poder tomar contacto con la naturaleza, lo cual hace que el turismo rural se convierta en una excelente alternativa para mejorar los ingresos de los campesinos.

Hasta este punto se ha dejado claro como la pobreza de las zonas rurales es preocupante y la brecha económica entre las zonas rurales y urbanas es profunda. De igual forma, se planteó que el turismo rural, puede ser una excelente alternativa en la busque de ingresos adicionales para el campo. Esta es precisamente la idea que se plantea, buscar establecer las pautas para la generación de una ruta agro turística para el sector cafetero del municipio de Gramalote.

Pero entender lo destacado de la propuesta, es importante recordar que el citado municipio sufrió una emergencia en su casco urbano debido a una falla geológica en el año 2010. Sus habitantes quedaron en situación de vulnerabilidad y diferentes entes gubernamentales han realizado proyectos para reconstruir el municipio y hacer que sea sostenible social y económicamente. En este sentido, el Plan de Desarrollo del municipio de Gramalote identificó las necesidades prioritarias que se deben de fortalecer, con la finalidad de generar programas que ayuden al sostenimiento económico y social del sector rural, teniendo en cuenta los ejes estratégicos identificados, aprovechando las potencialidades del municipio, donde el café es un punto fuerte ya que de acuerdo con el informe de empalme de la administración 2012-2015, en total hay 1.1157 hectáreas sembradas de café, de las cuales el 75\% corresponden a cafetales renovados con manejo básico y $25 \%$ a cafetales tradicionales de manejo rudimentario, de acuerdo al Comité Departamental de Cafeteros el rendimiento por hectárea es de 710 kilos de café pergamino seco (Acuerdo 012, 2016, p. 32).

Esto muestra la importancia que tiene el café con rubro económico del municipio, cuyos cultivos se encuentran ubicados en 24 veredas que se consolidan en cuatro sectores territoriales. Igualmente, el Departamento Nacional de Planeación (DNP, 2015) con los ciudadanos del municipio, identificaron algunas problemáticas que afectan la generación de ingresos y emprendimiento, siendo las principales: La pobreza rural, puesto que se detectó que muchos de las familias tienen ingresos inferiores al Salario Mínimo Mensual Vigente (SMLMV); el poco o nulo emprendimiento empresarial, por falta de fortalecimiento y capacitación a las asociaciones de productores; y por último, la ausencia de formación para el trabajo y el desarrollo humano, debido a la falta de oferta de educación Superior. 
Todos estos indicadores hacen pensar en la urgencia de buscar mecanismos que contribuyan de forma significativa a catapultar el desarrollo del municipio, ya que es prioritario hacer que las familias obtengan los ingresos suficientes para mejorar su calidad de vida. En este sentido, el Fondo de Adaptación en unión con la Federación Nacional de Cafeteros de Colombia y el municipio de Gramalote han propendido por la rehabilitación económica de los caficultores con el fin de aunar esfuerzos para la reactivación económica (Convenio No. CN-2012-1357, 2012, Clausula 1) con el proyecto de "Reactivación económica del municipio de Gramalote" en donde se buscó impactar a 435 familias caficultoras. Adicionalmente al proyecto en mención, también se había trabajado en el "resurgimiento productivamente bajo la sombra de café" en donde se renovaron 375 hectáreas cafeteras con variedades resistentes a la roya, beneficiando a 460 familias caficultoras.

Así mismo, tomando como base el documento Conpes No. 3803 (Departamento Nacional de Planeación, DNP, 2014), el cual establece como el Ministerio de Cultura presentó ante la UNESCO, la nominación del Paisaje Cultural Cafetero de Colombia a la lista de patrimonio mundial, inclusión que se hizo efectiva el 25 de junio de 2011 con la declaración de Valor Universal Excepcional. En consecuencia, mediante la Resolución 2079 (Ministerio de Cultura, 2011), se reconoce al Paisaje Cultural Cafetero de Colombia como Patrimonio Cultural de la Nación. Esto implica que se está buscando diversificar las fuentes de ingresos de los caficultores, no solo de Gramalote, sino de todo el país, con una atenuante importante, es un turismo que respeta las tradiciones agrícolas de la región, es decir, los ingresos que buscan incentivar el turismo agrícola, y adicionalmente, en la misma propuesta, mejorar la vida de estas familias, tradicionalmente aisladas y en muchas ocasiones en situaciones económicas precarias.

En este orden de ideas, es importante entender lo que se entiende por Paisaje Cultural Cafetero (PCC). Al respecto (Rincón, s.f.) apunta:

El PCC constituye un ejemplo sobresaliente de adaptación humana a condiciones geográficas difíciles sobre las que se desarrolló una caficultura de ladera y montaña. Se trata de un paisaje cultural en el que se conjugan elementos naturales, económicos y culturales con un alto grado de homogeneidad en la región, y que constituye un caso excepcional en el mundo. En este paisaje se combinan el esfuerzo humano, familiar y generacional de los caficultores con el acompañamiento permanente de su institucionalidad (párr. 4).

Esto implica que el paisaje cultural cafetero es el resultado de muchos años de esfuerzo de algunos pioneros que colonizaron zonas de difícil acceso y los convirtieron en asentamientos humanos que han ido pasando de generación en generación. Como afirma la ya citada Rincón (s.f.):

De esta manera se ha desarrollado una caficultura basada en la pequeña propiedad, que ha demostrado su sostenibilidad en términos económicos, sociales y ambientales, y que ha posicionado su producto como uno de los mejores cafés del mundo. Este modelo social y económico ha configurado una región con un alto grado de unidad cultural, expresada en un patrimonio cultural material en el que se destacan las técnicas constructivas tanto de los asentamientos urbanos como de las viviendas cafeteras rurales, así como un patrimonio cultural inmaterial en el que se expresa el vínculo de la población con el cultivo por medio de fiestas, carnavales y celebraciones (párr. 5). 
Sin embargo, los vaivenes del mercado internacional del grano, hacen que, en por diversas circunstancias el mono cultivo del café, no de los ingresos suficientes para tener una buena calidad de vida, ya que muchos de estos pequeños productores solo consiguen los recursos necesarios para la supervivencia. En el caso específico del municipio del Gramalote (Norte de Santader, Colombia), la situación es mucho más difícil, debido a la catástrofe geológica anteriormente mencionada y donde quedó destruido "el 94\% de casco urbano" (Acuerdo 012, 2016, p. 2). Sin embargo, es importante recordar que Gramalote es un municipio de vocación agrícola en el cual predominan las fincas dedicadas al cultivo de café, caña, banano y cítricos, así como la ganadería. Así mismo, el intercambio de bienes y servicios entre los habitantes del casco urbano y el sector rural era muy importante para la economía del municipio. Al igual que municipios aledaños como Lourdes, Santiago, Villacaro y el municipio de Salazar de las Palmas (Alcaldía Municipal de Gramalote en Norte de Santander, 2015).

\section{AnteCEDEnTES}

\section{Turismo Histórico - Cultural}

El turista actual se ha vuelto más frecuente su preferencia por pasar sus vacaciones en lugares que le reconforten en un todo, ya sea tanto física como intelectualmente. Por esto, la demanda turística crece hacia destinos poco tradicionales, cada vez con mayor regularidad, el turista tiene como objetivo el turismo históricocultural.

Este tipo de turismo llevará, entonces, a que los destinos turísticos deberán afrontar el gran desafío de revalorizar su Patrimonio Histórico, Cultural y Natural en pos de lograr que éstos se transformen en parte de los atractivos que busca el turista y permitan a este aumentar los pernoctes y gastos diarios promedio realizados en los destinos.

Se trata de revalorizar no tan solo un museo, paisajes, obras de arte, sino también los hechos históricos ocurridos en la localidad, industrias que se encuentran en la actualidad funcionando o en algún momento participaron en el desarrollo local, las comidas, fiestas populares del lugar o la región, entre otras muchas cosas. Para interiorizarse más de este tipo de turismo.

\section{Turismo rural}

Es una actividad turística que se realiza en un espacio rural, habitualmente en pequeñas localidades (menores a los 1.000 o 2.000 habitantes) o fuera del casco urbano en localidades de mayor tamaño. Las instalaciones suelen ser antiguas masías y caseríos que, una vez rehabilitados, reformados y adaptados, suelen estar regentados familiarmente, ofreciendo un servicio de calidad, en ocasiones por los mismos propietarios. El agroturismo, el turismo ecológico y el enoturismo son algunas de las modalidades que, de acuerdo a sus características, pueden incluirse dentro del turismo rural. 


\section{Turismo de aventura}

El turismo de aventura es dirigido para todos los turistas, pero en especial para aquellos que les gusta combinar sus actividades con el aire libre.

Este tipo de turismo también se relaciona directamente con el deporte de aventura o de riesgo, donde la gente tiene por objetivo pasar momentos de adrenalina a costo de un porcentaje del mismo.

El turismo de aventura tiene como objetivo principal el fomento de las actividades de aventura en la naturaleza. Es el hecho de visitar o alojarse en zonas donde se pueden desarrollar los llamados deportes de aventura o turismo activo.

La diferencia del turismo de aventura y deportes del turismo de aventura, estaría en que para la segunda actividad hace falta una preparación mínima y un equipo apropiado, como lo es practicar rappel, escalada, carreras de aventura o montañismo.

\section{Turismo ecológico}

Es una nueva tendencia del Turismo Alternativo diferente al Turismo tradicional. Es un enfoque para las actividades turísticas en el cual se privilegia la sustentabilidad, la preservación, la apreciación del medio (tanto natural como cultural) que acoge y sensibiliza a los viajantes. Aunque existen diferentes interpretaciones, por lo general el turismo ecológico se promueve como un turismo "ético", en el cual también se presume como primordial el bienestar de las poblaciones locales, y tal presunción se refleja en la estructura y funcionamiento de las empresas, y cooperativas que se dedican a ofrecer tal servicio.

\section{Municipio de Gramalote}

En este punto queda aclarada la situación del sector rural colombiano y en específico la del municipio de Gramalote, que siendo una región tradicionalmente agrícola forma parte de un grupo de ciudadanos sometidos a una serie de dificultades que se debe propender por superar. Pero para que esto se logre es necesario diversificar las actividades económicas de la región, y el agro turismo, que ha ido tomando relevancia en el país, en especial en el sector cafetero, es una alternativa interesante que puede ser viable en la búsqueda de la superación de las brechas económicas y sociales del sector agrario. Igualmente, el cultivo del grano de café ha servido de sustento a un importante grupo poblacional campesino y se ha convertido en el producto bandera de la nación, constituyéndose, no solo en fuente de ingreso por su venta, sino que ha sido una forma de promocionar el país desde el punto de vista turístico y comercial. Es por ello que importantes regiones cafeteras ha ido incorporando entre sus actividades el desarrollo del turismo agrícola, pues la belleza natural de estas regiones tiene un enorme potencial para el desarrollo de la actividad, en especial para los extranjeros que ven en la forma artesanal de producción algo exótico digno de ser conocido. 
Lo apuntado, justica claramente la importancia de desarrollar proyectos agroturísticos basados en el café. En el caso particular de Gramalote, el producto es un baluarte importante de su economía, y, por lo tanto, un buen punto de partida para el desarrollo del turismo agrícola. En este orden de ideas cabe preguntarse ¿Cuál es la viabilidad financiera para desarrollar una ruta agro turística del sector cafetero del municipio de Gramalote, Norte Santander?

\section{Metodología}

Para el desarrollo del proyecto de investigación se tuvo en cuenta el enfoque cuantitativo. Este tipo de investigación busca información cuantitativa orientada por conceptos empíricos medibles, derivados de los conceptos teóricos con los que se construyen las hipótesis conceptuales. El análisis de la información recolectada tiene por fin determinar el grado de significación de las relaciones previstas entre las variables. (Monje, 2011).

En este sentido la investigación cuantitativa busca establecer una relación causaefecto haciendo uso de datos medibles y sigue un proceso hipotético-deductivo el cual inicia con la formulación de las hipótesis derivadas de la teoría, continúa con la operacionalización de las variables, la recolección, el procesamiento de los datos y la interpretación. Los datos empíricos constituyen la base para la prueba de las hipótesis y los modelos teóricos formulados por el investigador (Monje, 2011).

El enfoque cuantitativo fue relevante para la investigación al compilar información sobre el nivel de producción y económico de las fincas, para así poder entender la factibilidad de la propuesta. En un sentido similar, el proyecto se orientó en el método de investigación descriptiva que busca trabajar sobre realidades de hecho y su característica fundamental es la de presentar una interpretación correcta (Arias, 2012) de la realidad estudiada, que es precisamente lo que se buscó en el proceso, conocer la realidad económica y de producción de las fincas investigadas, de modo de tener herramientas de juicio suficientes para verificar hasta qué punto es posible desarrollar la ruta agro turística.

De otro lado, el tipo de investigación fue de campo, que de acuerdo con Arias (2012) "es aquella que consiste en la recolección de datos directamente de los sujetos investigados, o de la realidad donde ocurren los hechos (datos primarios), sin manipular o controlar variable alguna" (p. 31); lo cual implica tomar la información, pero sin alterar las condiciones existentes, lo que hace que esta forma de investigar se considere no experimental.

Al ser una investigación de corte cuantitativo, es importante establecer una hipótesis. Dicha hipótesis fue: ¿Es viable económicamente el desarrollo de una ruta agro turística, basada en el café, para el municipio de Gramalote, Norte de Santander? Asimismo, la hipótesis nula establece la no es viabilidad económica del desarrollo de una ruta agro turística, basada en el café, para el municipio de Gramalote, Norte de Santander.

Es por ello que el trabajo fue guiado por los siguientes aspectos: 
El primero, caracterizar socioeconómica y productivamente las fincas cafeteras del municipio de Gramalote, Norte Santander, con la finalidad de entender las realidades que se viven en ellas. En segundo lugar, identificar las apuestas de valor agregado de la producción de las fincas cafeteras del municipio de Gramalote, Norte Santander. Una vez identificado este valor agregado, se procedió a evaluar la viabilidad financiera de la ruta turística del café en el municipio de Gramalote, Norte de Santander, así poder proponer una ruta turística del café en el municipio de Gramalote Norte, Santander.

\section{Resultados}

Para dar cumplimiento al primer aspecto de caracterizar socioeconómica y productivamente las fincas cafeteras del municipio de Gramalote Norte Santander (Colombia), se realizaron encuestas a cinco fincas de la región, configurados por 5 cuestionarios que buscaron entender a mayor profundidad las características de las fincas.

Con referente al primer cuestionario sobre las características generales de la unidad productiva, de su análisis se encontró que el promedio de edad de las personas que son la cabeza visible de la unidad productiva fue de 47 años, lo cual implica que son personas adultas, con la suficiente experiencia en su manejo. Otro aspecto sobre el que se indago es el referente a la propiedad o no de la tierra, concluyéndose que todas las fincas son propias, es decir, que sus habitantes son dueños de las tierras.

De otro lado, en cuanto al tamaño de los grupos familiares estos, el promedio de miembros de las mismas es de 7.8, situación normal en la vida del campo. En cuanto a la edad de los habitantes, la gran mayoría de las personas se encuentran entre los 14 y 20 años, cifra que coincide con los resultados arrojados por el último censo. Un dato importante es el que tiene que ver con el estudio de los hijos, encontrándose que en todos los núcleos familiares investigados estos estudian, muchos de ellos carreras profesionales que tiene que ver con la producción del campo.

Otro aspecto importante, fueron las actividades productivas, destacándose la producción de café, seguida de la de cítricos y caña de azúcar. En cuanto a lo pecuario, sobresale la cría de cerdos y pollos, siento esta actividad, más para el consumo doméstico que para el comercio.

Al hacer referencia a la operación de las fincas, estas trabajan en su mayoría los doce meses del año. De igual forma, cabe destacar que ninguna de las fincas ha sido legalizada ante la DIAN como unidad productiva, situación que seguramente dificulte el acceso a créditos. Igualmente, el promedio de personas por finca que estarían disponibles para atender la ruta, este es de 3,5 personas, lo que muestra que hay un verdadero interés por el proyecto.

El segundo cuestionario, indagó sobre las características físicas y de acceso a los predios. En cuanto al tamaño de los terrenos, en su mayoría son parcelas no superiores a las 7 hectáreas. Sin embargo, también existen terrenos de gran extensión. Un rasgo sobresaliente, es que las zonas construidas son relativamente pequeñas con respecto al tamaño de los predios. 
Es importante resaltar que el acceso a las fincas son tierras planas, pero sin pavimentar, lo que hace que los transportes más úsales sean los camperos y las motocicletas. De igual forma, se verifico que los productores encuestados se encuentran asociados, en especial a la Asociación Nacional de Cafeteros.

La principal especie de grano cultivado es la Castilla variedad Colombia. De igual forma se pudo establecer que una de las fincas desarrolla cultivos orgánicos. Este aspecto es importante, ya que, sobre todo los extranjeros, buscan productos libres de agroquímicos.

El tercer formato, busco caracterizar los recursos naturales, donde se destaca la biodiversidad especialmente de la flora. Una de las fincas es particularmente atractiva, debido a que posee más de cuarenta especies flores. Asimismo, los animales más destacados de la zona son los colibrís y los azulejos, especies de pájaros de colores atractivos, abundantes en la región.

Un aspecto importante, es el alto grado de conservación en que se encuentran las fincas, lo que permite entender, que, si bien se requieren mejoras locativas, en realidad es que lo debe buscarse es ampliar las zonas construidas. Respecto a las actividades turísticas que pueden darse, estas son diversas y tienen que ver con la exploración de la vida en el campo, es decir, ir a los cafetales, colaborar en la alimentación de los animales, dar paseos a caballo, ir a miradores para la toma de fotografías, entender el proceso de producción del café, hacer paseos ecoturísticos y acampar. A continuación, se muestran algunas fotografías de los lugares visitados, donde se puede corroborar lo apuntado.

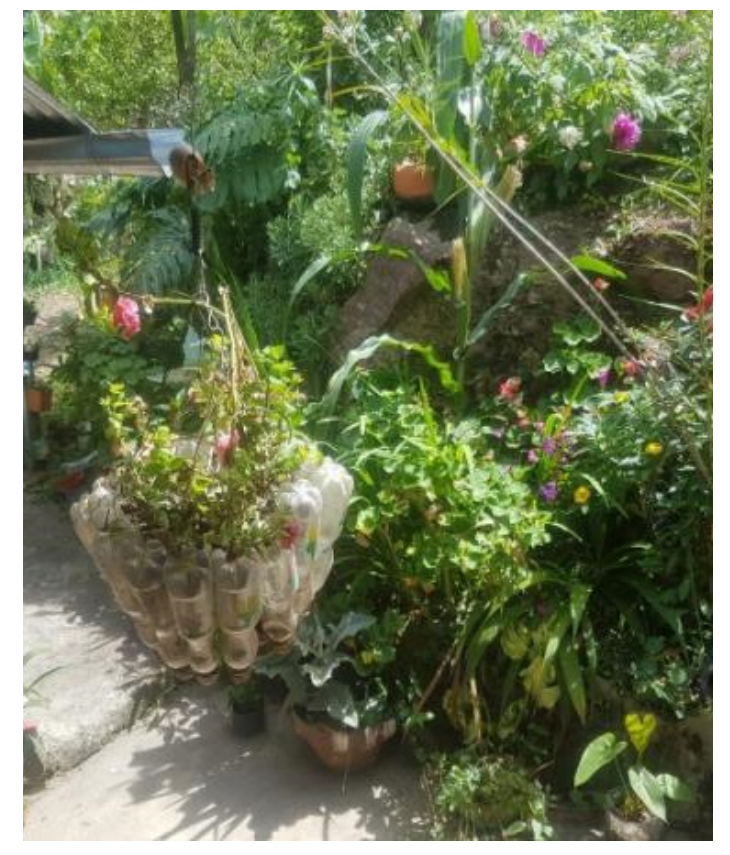

Figura 1. Especies de flores, Finca las margaritas. Fuente: Elaboración propia.

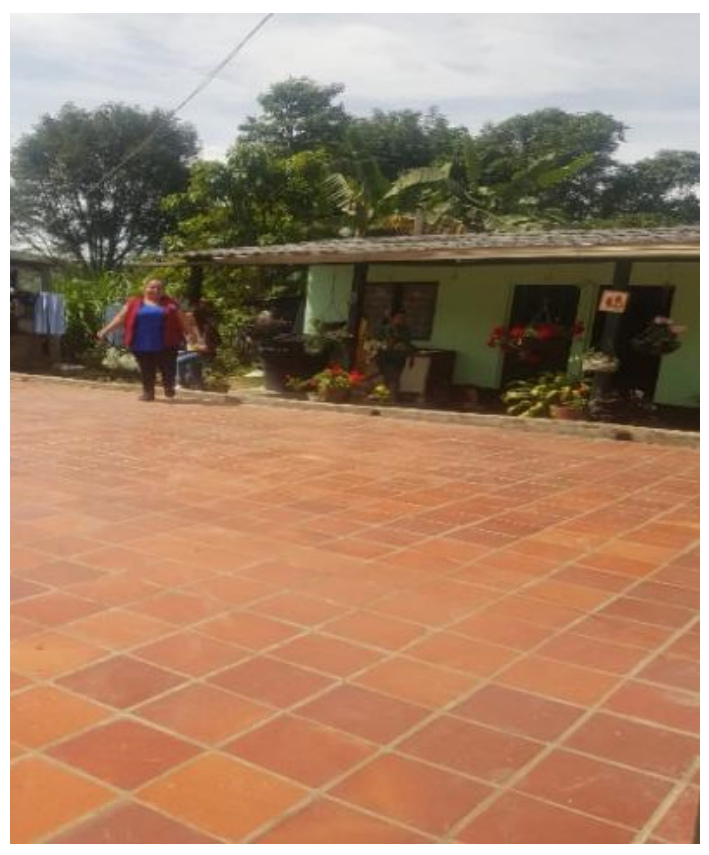

Figura 2. Foto patio beneficio de café (Finca Las Margaritas). Fuente: Elaboración propia. 


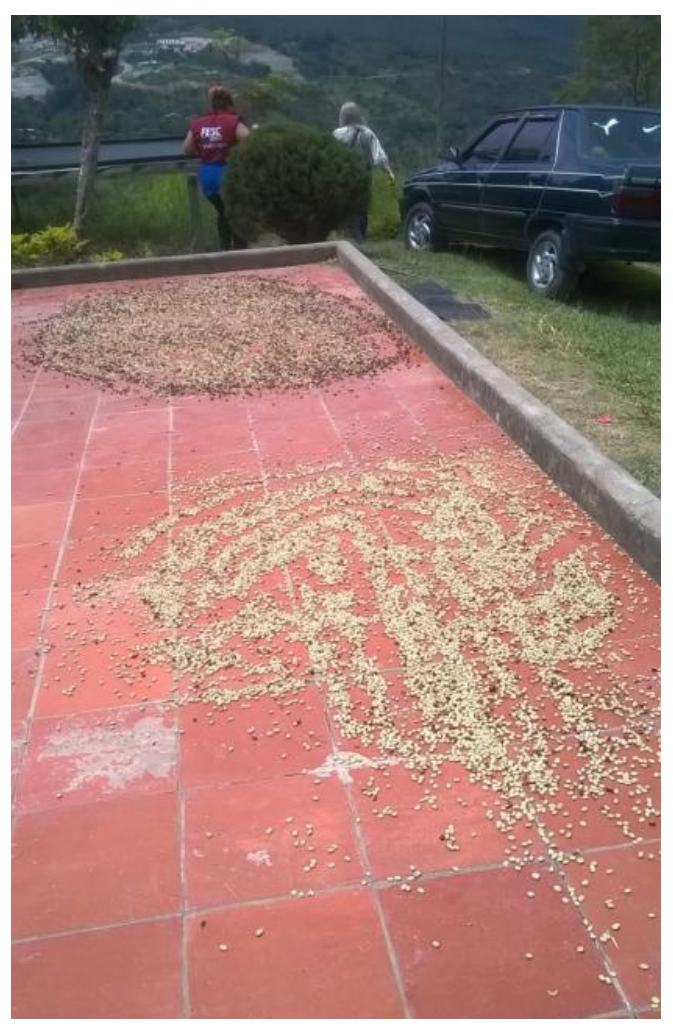

Figura 3. Foto de secado tradicional de café (Finca Los Naranjitos). Fuente: Elaboración propia.

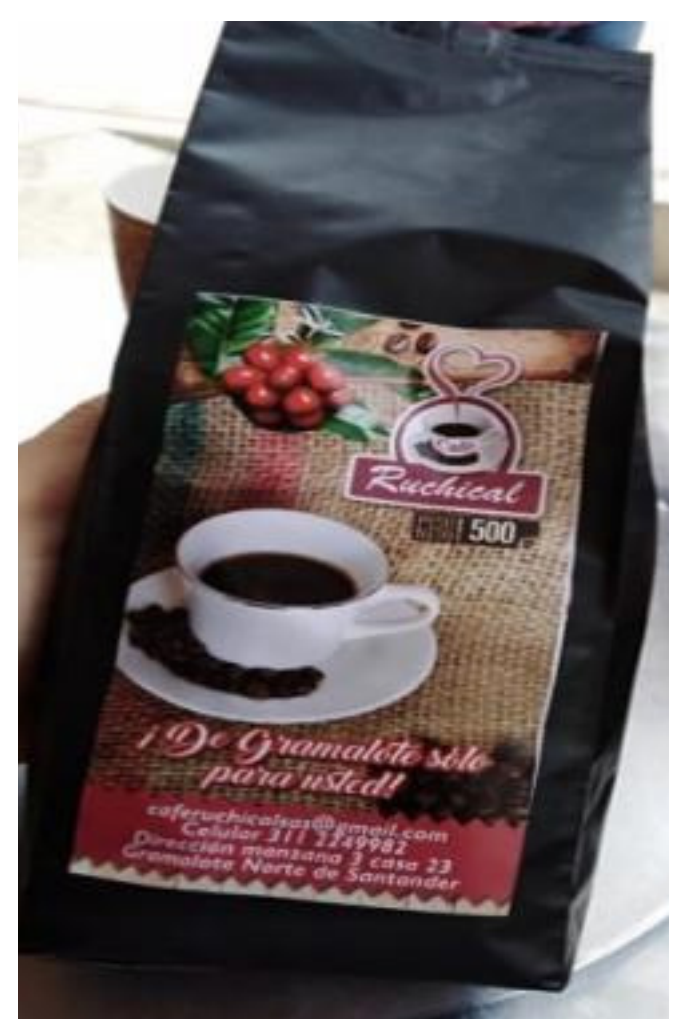

Figura 4. Foto café orgánico (Finca El Ruchical). Fuente: Elaboración propia.

El cuarto formulario analizado, hace referencia a las características del alojamiento, es decir, entender qué tipo de construcciones son las disponibles para el desarrollo de la ruta turística. La disponibilidad del alojamiento, es buena, además las propiedades están construidas en ladrillo, lo que es relevante en términos de calidad de las habitaciones, pero resta atractivo por no ser construcciones tradicionales. En el mismo sentido, se encontró que todas las fincas tienen servicios sanitarios, así como de cocina tanto de gas, en algunos casos de biomasa, al igual que el uso tradicional a leña.

El servicio de agua en su mayoría proviene de las nacientes de agua, lo que por sí mismo es un atractivo. Igualmente, algunas de las fincas ofrecen comidas tradicionales de la región, donde se destaca el "chocheco", que es una variedad de plátano muy apreciado en el sector. En cuanto a la celebración festivas sobresale la celebración del aniversario de fundación del pueblo (27 de noviembre) y la Epifanía del Señor o fiesta de los reyes magos ( 6 de enero). También es importante mencionar que en la región circulan una importante cantidad de leyendas, que son historias trasmitidas por tradición oral y que hacen referencia a espantos. Otro aspecto importante tiene que ver con la existencia de maquinarias antiguas de uso tradicional en la industria del café, tales como molinos, despulpadoras, como se puede apreciar en el siguiente material fotográfico recopilado por la autora.

Para dar concluida la caracterización se abordará un tópico importante, el referente a los propósitos que tienen los propietarios, en relación a los posibles servicios que desean prestar a los turistas. Estos se resumen en la Tabla 1. 


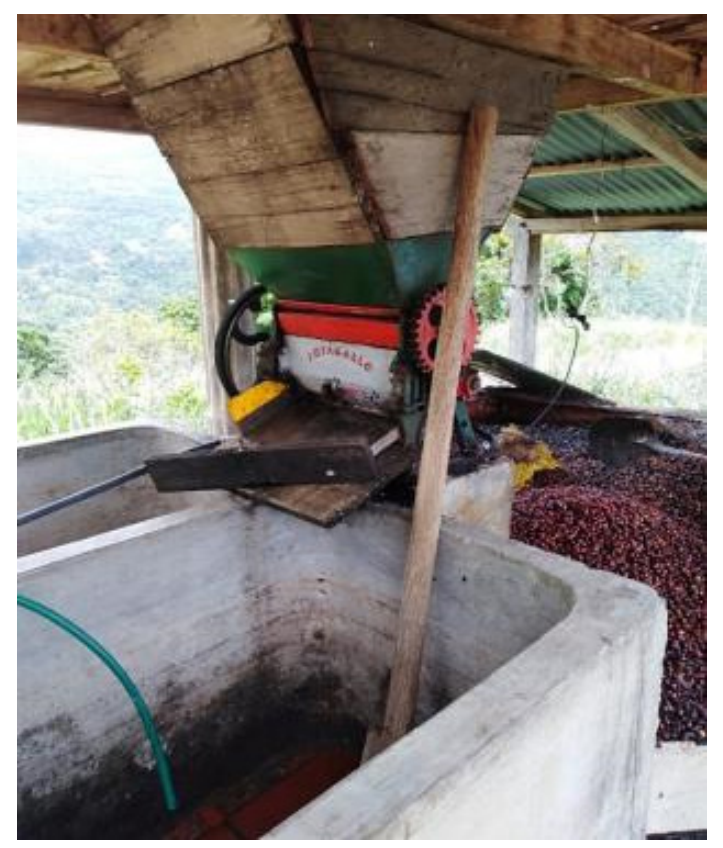

Figura 5. Despulpadora de grano (Finca los Naranjitos). Fuente: Elaboración propia.

TABLA 1.

Propósitos turísticos de las fincas

\begin{tabular}{llll}
\hline \multicolumn{1}{c}{ Servicio } & si & no & no contesta \\
\hline Alojamiento & 2 & 3 \\
Restaurante (alimentación y bebidas) & 3 & 2 \\
Museo & 3 & 2 \\
Cultivo de café & 3 & 2 \\
Paseo a caballo & 2 & 3 \\
Deportes de aventura & & \multicolumn{2}{c}{} \\
Casas de descanso & 3 & 2 \\
Otros servicios turísticos & & \multicolumn{2}{c}{5} \\
\hline
\end{tabular}

Fuente: Elaboración propia.

Se destaca de la Tabla 1 que se desea prestar servicio de restaurante, las casas de descanso y servir de museos. La realidad es que algunos de los encuestados no contesto completamente este apartado, pero se puede inferir que existe la voluntad de adherirse al proyecto, puesto que dentro de este mismo test se preguntó por las expectativas que se tienen con respecto a la idea de la ruta agro turística, se pudo evidenciar que existe voluntad, que se demuestra en la idea de ser capacitados para este tipo de actividad. Además, la mayoría de los encuestados tiene algún proyecto de mejorar que no se ha llevado a cabo, debido a la falta de recursos.

En el cumplimiento del segundo objetivo, identificar las apuestas de valor agregado de la producción de las fincas cafeteras del municipio de Gramalote (Norte de Santander). Del análisis precedente, se puede afirmar que las fincas investigadas presentan las características físicas que permite afirmar que se tienen las condiciones necesarias para el desarrollo de la ruta agro turística del café. Unido a esto, existe 
un alto valor agregado, debido a la amabilidad y nobleza de las gentes del sector para dar atención a los foráneos, al igual que el conocimiento de la actividad agrícola que se centra evidentemente en el café, que es un oficio tradicional en la región. De otro lado, se tiene la voluntad de mejora constante, unido al conocimiento de las tradiciones de este sector del departamento, especialmente en lo que tiene que ver con los platos típicos, aspectos culturales como la música, las leyendas, el folclor. Asimismo, los aspectos históricos, tales como la historia de las fincas, el conocimiento de los procesos necesarios para la producción del grano de café y su transformación. Es claro, que desde lo cultural y las condiciones de geográficas y de infraestructura de las fincas, todo se encuentra dado para el desarrollo del proyecto.

Una vez cumplidos los dos objetivos precedentes, queda un tercero, que tiene que ver con la viabilidad financiera para la implementación de ruta agro turística del café de Gramalote. En este sentido, continuación se presentan los análisis económicos de los mismos.

En primer lugar, las personas que deseen acceder a los posibles planes turísticos (Tabla 2), puede elegir el de su preferencia, con el propósito que le permita conocer cada una de las fincas cafeteras que conforman la ruta, para que pueda conocer los hermosos países y la herencia cultural de cada una de estas. De la misma manera podrán elegir si desean alojarse en las fincas disponibles para alojamiento, o si por el contrario desea pasar la noche bajo la luz de la luna a cielo abierto (camping). Además, con el propósito de que los visitantes conozcan el casco urbano de Gramalote, tendrán la opción de hospedarse en viviendas disponibles para este propósito en el nuevo casco urbano (Miraflores); esto con el mayor deseo de que los visitantes vivan la mejor experiencia del campo, que se desconecten de todas sus cargas y puedan disfrutar de su estadía en el municipio de Gramalote (Norte de Santander).

TABLA 2.

Posibles planes turísticos

\begin{tabular}{|c|c|c|}
\hline Plan & Características & Valor (COP) \\
\hline Plan 1 & $\begin{array}{l}\text { - } 1 \text { día } \\
\text { - Recorrido por el antiguo Gramalote (Ruinas) } \\
\text { - Recorrido por las fincas cafeteras y cada uno de los procesos del café } \\
\text { - Desayuno y almuerzo }\end{array}$ & $\$ 180.000$ \\
\hline Plan 2 & $\begin{array}{l}\text { - } 2 \text { días } \\
\text { - Recorrido por el antiguo Gramalote (Ruinas) } \\
\text { - Recorrido por las fincas cafeteras y cada uno de los procesos del café } \\
\text { - Desayunos y almuerzos } \\
\text { - Estadía en finca/casco urbano/camping }\end{array}$ & $\$ 230.000$ \\
\hline Plan 3 & $\begin{array}{l}\text { - } 3 \text { días } \\
\text { - Recorrido por el antiguo Gramalote (ruinas) } \\
\text { - Recorrido por las fincas cafeteras y cada uno de los procesos del café } \\
\text { - Desayunos y almuerzos } \\
\text { - Estadía en finca/casco urbano/camping } \\
\text { - Deporte extremo(parapente) }\end{array}$ & $\$ 320.000$ \\
\hline
\end{tabular}

Fuente: Elaboración propia. 
En segundo lugar, se muestra la proyección de ventas a 10 años, de cada uno de los planes, con una proyección anual de aumento de servicios del 3,5\% y un 10\% de incremento en el valor de cada plan (Figura 6).

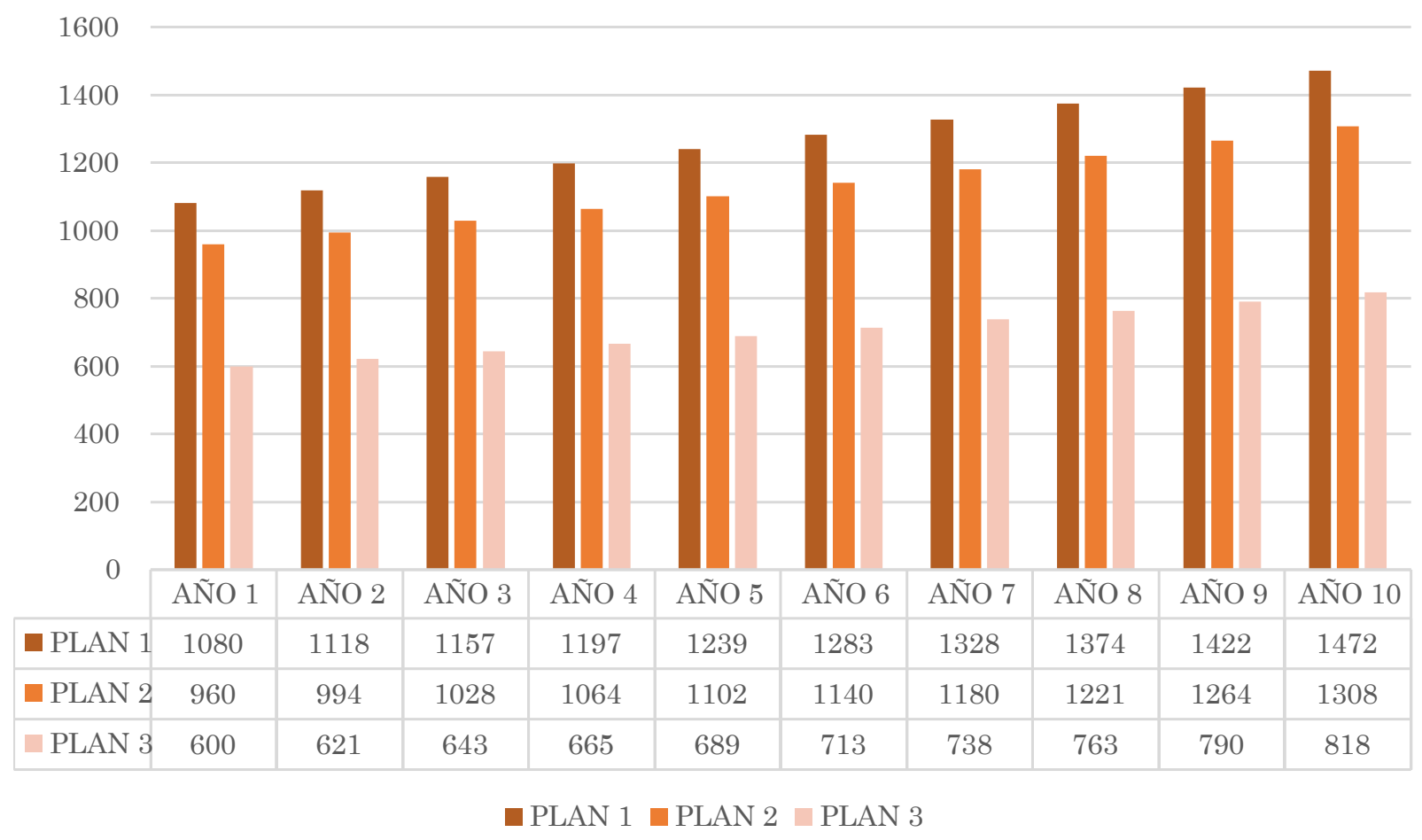

Figura 6. Proyección de ventas anuales por plan a 10 años. Fuente: Elaboración propia.

Como ya se afirmó se hizo una proyección de crecimiento de venta de planes del $3,5 \%$ por año, por lo que la cantidad de ventas de los tres planes comenzará con 2640 en el primer año para llegar hasta 3598 en el 10, lo que significa un aumento de 850 productos en una década.

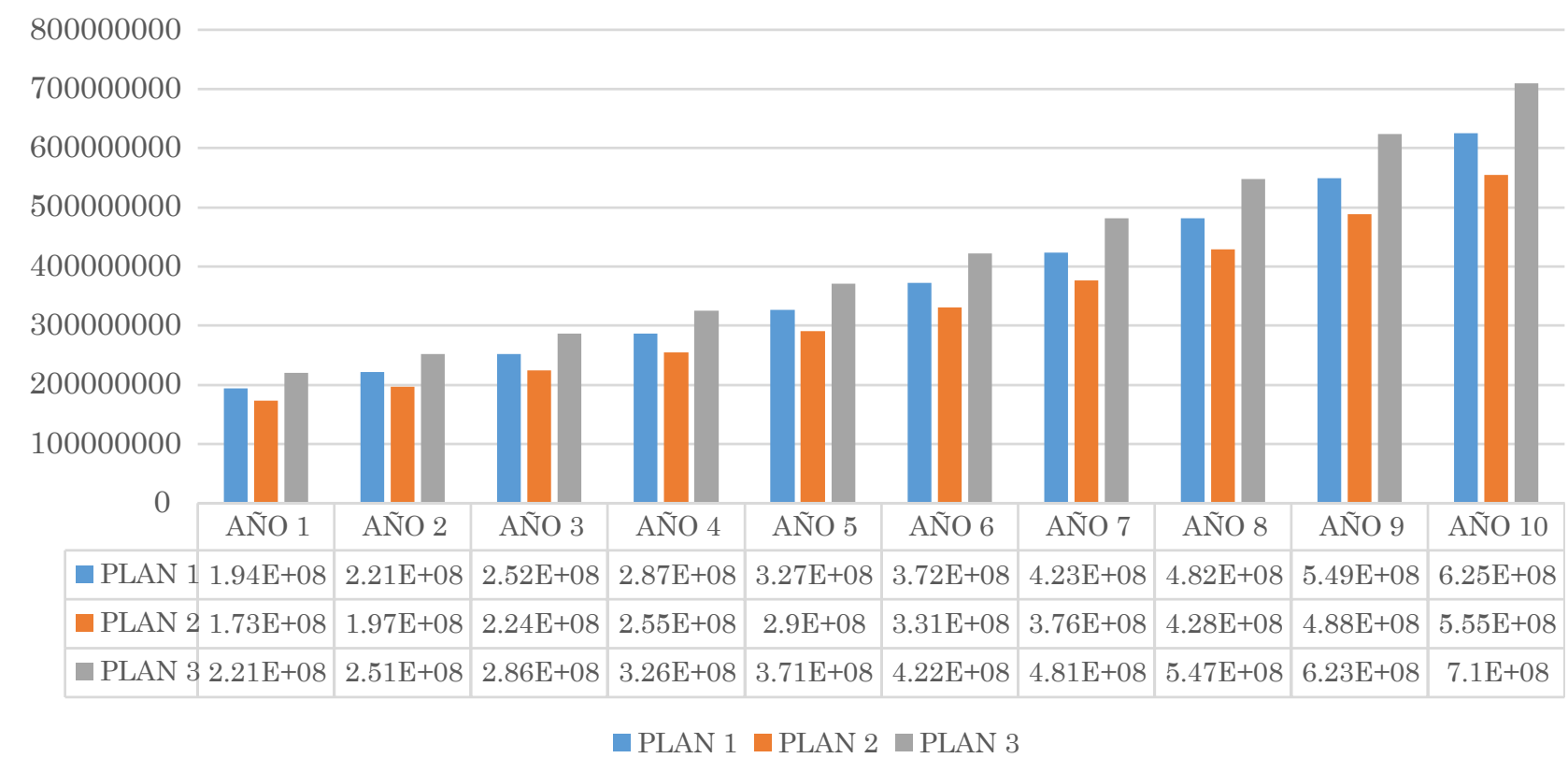

Figura 7. Proyección de ingresos a 10 años por plan.

Fuente: Elaboración propia. 
De acuerdo con esta proyección de ventas, los ingresos proyectados deberán pasar de $\$ 588.000 .000$ antes del IVA, y para el primer año serán de $\$ 1.889 .620 .712$, lo cual significa un aumento de ingresos de más de 1.000 millones de pesos en los primeros 10 años, de donde se pude concluir que los ingresos brutos promedio anuales deberán crecer 100 millones al año, es decir, un negocio de \$11.287.604.195 antes de impuestos; mismos que le pueden generar al estado por concepto de IVA unos \$2.144.644.797 (Figura 7). Sin embargo, como ya se ha apuntado, se requieren una serie de adecuaciones y de dotación para poder iniciar con el servicio. En este sentido se presenta una proyección de los gastos necesarios en estos dos rubros (Tabla 3).

TABLA 3.

Inversión proyección de gastos para adecuación de activos fijos iniciar operaciones

\begin{tabular}{lll}
\hline \multicolumn{1}{c}{ Rubro } & \multicolumn{1}{c}{ Valor unitario } & \multicolumn{1}{c}{ Valor total } \\
\hline Adecuaciones & $\$ 150.000 .000$ & $\$ 150.000 .000$ \\
Toallas, ropas de cama, cortinas & $\$ 8.000 .000$ & $\$ 8.000 .000$ \\
Loza(platos, vasos, cubiertos, etc.) & $\$ 5.000 .000$ & $\$ 5.000 .000$ \\
Carpas de camping y sliping & $\$ 5.000 .000$ & $\$ 5.000 .000$ \\
Sillas, mesas, camas & $\$ 12.000 .000$ & $\$ 12.000 .000$ \\
& Total & $\$ 180.000 .000$ \\
\hline
\end{tabular}

Fuente: Elaboración propia.

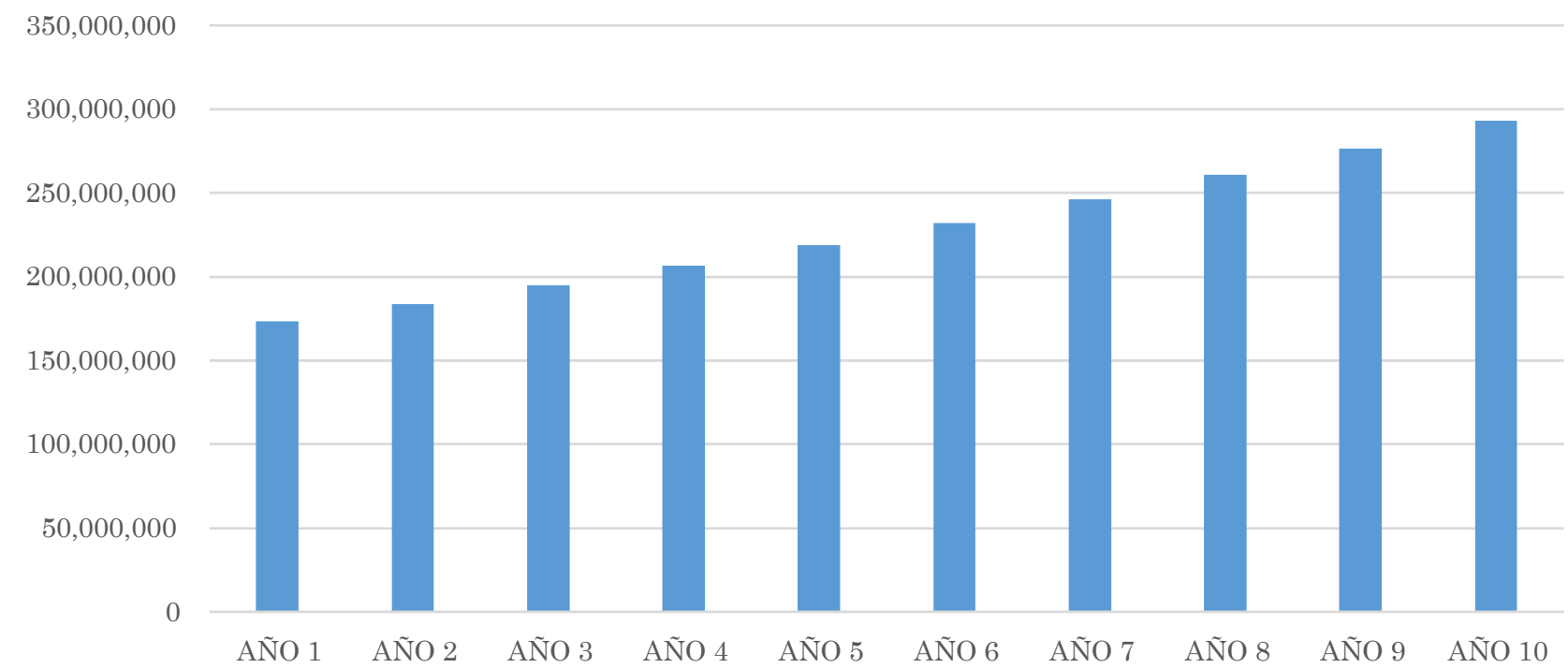

Figura 8. Proyección de gastos anuales por nómina.

Fuente: Eaboración propia.

Es claro que para el desarrollo de la ruta turística se hace necesario la contratación de personal especializado que contribuya en la optimización de los servicios. Es el por ello que se proyecta la contratación de 3 guías, 2 guías bilingües, 1 chef, un ayudante de cocina, una persona para aseo general y una secretaria. Los cálculos de nómina se proyectaron tiene en cuenta un incremento anual del 6\%. La proyección del gasto de empleados se presenta en la Tabla 4, teniéndose en cuenta que, para el personal no especializado, es decir, los 3 guías, la aseadora, el ayudante de cocina y la secretaria, 
se proyectó sobre un sueldo mínimo mensual, mientras que para los guías bilingües se consideró un sueldo de un millón, y para el chef de millón doscientos mil.

La proyección para los gastos de administración se proyectó sobre un aumento del 9\% anual, considerando los siguientes rubros: Mantenimiento, gas natural, servicio de electricidad, teléfono e internet, arriendo, implementos de Seguridad Industrial, dotación Personal Operativa, implementos de Aseo, consultoría y papelería (Tabla 4).

TABla 4.

Proyección gastos de administración

\begin{tabular}{ll}
\hline & \multicolumn{1}{c}{ Costo } \\
\hline Año 1 & 40.560 .000 \\
Año 2 & 44.210 .400 \\
Año 3 & 48.189 .336 \\
Año 4 & 52.526 .376 \\
Año 5 & 57.253 .750 \\
Año 6 & 62.406 .588 \\
Año 7 & 68.023 .180 \\
Año 8 & 74.145 .267 \\
Año 9 & 80.818 .341 \\
Año 10 & 88.091 .991 \\
\hline
\end{tabular}

Fuente: Elaboración propia.

En la proyección de los gastos de venta se tuvo en cuenta un aumento del 9\%, empezando el año 1 con un valor de $\$ 30.000 .000$ hasta alcanzar los $\$$ 65.156.798. De igual forma se consideró que para dar inicio al negocio se necesita tener una provisión para nóminas y gastos para 3 meses, lo que implica que se requieren $\$ 84.220 .181$, incluyendo la compra de algunos muebles y un valor para inicio de labores.

TABLA 5.

Proyección de utilidades netas

\begin{tabular}{ll}
\hline & \multicolumn{1}{c}{ Utilidad } \\
\hline Año 1 & 299.794 .064 \\
Año 2 & 346.690 .283 \\
Año 3 & 399.603 .012 \\
Año 4 & 459.883 .407 \\
Año 5 & 528.570 .110 \\
Año 6 & 606.847 .253 \\
Año 7 & 696.064 .759 \\
Año 8 & 797.761 .463 \\
Año 9 & 913.691 .449 \\
Año 10 & 1.045 .854 .063 \\
\hline
\end{tabular}

Fuente: Elaboración propia. 
Si bien, los dueños de las fincas deberán hacer un aporte, es necesario la tramitación de un préstamo por un valor de $\$ 67.376 .144$. Este dinero tiene un costo del $29 \%$ anual y debe cancelarse en los diez años de operación proyectados. De acuerdo con los cálculos realizados el negocio es rentable, siempre y cuando se cumplan las metas de venta establecidas, generando las utilidades netas, después del descuento de la reserva, que se muestran en la Tabla 5.

Esto implica que el negocio debería producir $\$ 6.094 .759 .862$ en los 10 años proyectados, mostrando que el negocio es altamente rentable. En definitiva se puede afirmar que la ruta agro turística del café en el municipio de Gramalote (Norte de Santander, Colombia) requiere una inversión de $\$ 234.220 .181$, generando la posibilidad de realizar un crédito equivalente al $80 \%$ del capital necesario y el $20 \%$ restante, sería aportado por los dueños de las fincas pertenecientes a la ruta; esta inversión está proyectada a 10 años, dando como resultado unos flujos de efectivos favorables en cada periodo, siempre y cuando los ingresos sean igual a los proyectados. Igualmente, los indicadores financieros de esta inversión arrojan resultados positivos, ya que el margen de utilidad en promedio es del 50\% y un nivel de endeudamiento que al año 10 será cero (0) y con una tasa interna de retorno de más del 100\% de la inversión.

\section{ConClusiones}

Del análisis realizado se puede concluir que la zona del municipio de Gramalote, tiene un excelente potencial para el desarrollo de una ruta agro turística, basada en el café, debido a que existen los elementos necesarios para que crear un verdadero atractivo turístico, desde las reservas naturales, así como la infraestructura de predios para su implementación. Este sector del departamento cuenta con excelentes paisajes, al igual que con una tradición cultural cafetera que puede ser atractiva para los turistas tanto locales como extranjeros.

En un sentido similar, los propietarios de fincas muestran la disposición para dar inicio a un negocio que puede convertirse en altamente rentable, dando dividendos a los habitantes de la región, como en la generación de ingresos por conceptos de IVA. Si bien se hace necesario realizar algunas adecuaciones, es posible a corto plazo dar viabilidad al proyecto, ya que la diversidad de flora y fauna, unido a una gastronomía propia y un acervo cultural enraizado y bien conservado por la tradición oral, fuentes propias de agua y accesibilidad, se establecen como puntos de partida importantes para dar impulso a una ruta turística que contribuya a diversificar los ingresos de los campesinos de la zona.

Desde el punto de vista financiero, se pudo comprobar que la ruta agro turística del café de Gramalote puede generar ganancias netas equivalentes al 50\% de la inversión realizada, situación que convierte al proyecto en factible, lo que permite afirmar que se acepta la hipótesis del investigador, es decir que es viable económicamente el desarrollo de una ruta agro turística, basada en el café, para el municipio de Gramalote (Norte de Santander). De ahí que se pude concluir que es recomendable dicha inversión, ya que cuenta con una excelente liquidez. 


\section{Reconocimientos y Agradecimientos}

Artículo de investigación y análisis prospectivo empresarial. Proyecto Apuesta de competitividad de la ruta agroturística del sector cafetero del municipio de Gramalote, Norte de Santander de los Grupos de Investigación: Competitividad y Sostenibilidad para el Desarrollo de la Universidad Libre (Cúcuta, Colombia) y Grupo de Investigación en Logística, Competitividad y Negocios Internacionales Grupo GILOCNI- de la Universidad Francisco de Paula Santander-UFPS (Cúcuta, Colombia), realizado como resultado de procesos de investigación.

\section{REFERENCIAS}

Alcaldía Municipal de Gramalote en Norte de Santander. Concejo municipal. (1 de junio de 2016). Plan de desarrollo "unidos por Gramalote 2016-2019”. [Acuerdo 012]. Disponible en http://www.gramalote-nortedesantander.gov.co/planes/ unidos-por-gramalote-20162019

Alcaldía Municipal de Gramalote en Norte de Santander. (2015). Documento de Análisis Situación de Salud 2015. ASIS 2015. [Online]. Disponible en http:// www.gramalote-nortedesantander.gov.co/

Arias, F. G. (2012). El proyecto de investigación. Introducción a la metodología científica. (6 ed.). Caracas: Episteme.

Kay, C. (2007). Pobreza rural en América Latina: teorías y estrategias de desarrollo. Revista mexicana de sociología, 69(1), 69-108. Recuperado de http://revistamexicanadesociologia.unam.mx/index.php/rms/article/view/6084

Monje, C. (2011). Metodología de la investigación cuantitativa y cualitativa. Neiva: Universidad Surcolombiana.

Pérez, M. E., Chumaceiro, A. y Acosta, I. (2019). Sostenibilidad social en el sector agroindustrial del estado Trujillo-Venezuela. Un constructo relevante para el desarrollo. Opción, 35(90), 100-149. Disponible en https://produccioncientificaluz.org/index.php/opcion/article/view/30460/31506

Pérez, S. F. (2010). El valor estratégico del turismo rural como alternativa sostenible de desarrollo territorial rural. Agronomía Colombiana, 28(3), 507-513. Disponible en https://revistas.unal.edu.co/index.php/agrocol/article/view/14688

República de Colombia. DNP. (2015). Plan nacional de desarrollo para Gramalote. [Online]

República de Colombia. DNP. (2014). Política para la Preservación del paisaje cultural Cafetero de Colombia. [Conpes 3803]. Recuperado de https://colaboracion. dnp.gov.co/CDT/Conpes/Econ\%C3\%B3micos/3803.pdf

República de Colombia. Fondo de adaptación. Federación Nacional de Cafeteros. Municipio de Gramalote. (2012). Convenio No. CN-2012-1357. [Online]. Recuperado de http://fondoadaptacion.gov.co/download/ConvenioFA_Federacion_Cafeteros_Mpio_Gramalote(2).pdf 
República de Colombia. Ministerio de Cultura. (7 de octubre de 2011). Por la cual se reconoce al Paisaje Cultural Cafetero de Colombia como Patrimonio Cultural de la Nación. [Resolución 2079]. Diario Oficial: 48.634. Recuperado de https:/www.mincultura.gov.co/prensa/noticias/Documents/Patrimonio/5.\%20 Resoluci\%C3\%B3n\%202079\%20de\%202011\%20PCC.pdf

Rincón, C. (s.f.). Paisaje Cultural Cafetero. [Online]. Mincultura. Disponible en http://www.mincultura.gov.co/areas/patrimonio/investigacion-y-documentacion/ politicas-planes-y-programas/Paginas/Paisaje-Cultural-Cafetero.aspx

Semana. (agosto, 2015). Una dura radiografía del campo colombiano. semana.com. Recuperado de https://www.semana.com/economia/articulo/campo-colombianoen-la-pobreza/438618-3

BIODATA

Johanna Milena Mogrovejo Andrade es docente de la Universidad Libre (Cúcuta, Colombia) con doctorado en Estudios Políticos de la Universidad Externado de Colombia. https://orcid.org/0000-0002-9694-3382

Cesar Augusto Panizo Cardona es docente Tiempo Completo de la Universidad Francisco de Paula Santander (Cúcuta, Colombia) con magíster en Administración de Negocios - MBA de la Universidad de Medellín - UdeM (Medellín, Colombia). https://orcid.org/0000-0002-9507-4963

Marilyn López Echeverry estudia Contaduría Pública en la Universidad Libre (Cúcuta, Colombia). Auxiliar de investigación con fines de homologación. Auxiliar contable para la firma de contadores DSC Desarrollando Soluciones Contables. https://orcid.org/0000-0003-0984-3613 